\title{
Forensic Pharmacy Practice: New Initiatives in Saudi Arabia
}

\author{
Yousef Ahmed Alomi*, (iD Bsc. \\ Pharm, Msc. Clin pharm, BCPS, BCNSP, DiBA, \\ CDE, Critical Care Clinical Pharmacists, TPN \\ Clinical Pharmacist, Freelancer Business \\ Planner, Content Editor, and Data Analyst, \\ Riyadh, SAUDI ARABIA
}

Rehab Sultan Najmi, Bsc. Pharm, King Khalid University Abha, SAUDI ARABIA. Abeer Hussin Almasoudi, BSC. Pharm, Director, Administration of Research and Studies, Ministry of Health, Tabuk, SAUDI ARABIA.

\section{Correspondence:}

Dr. Yousef Ahmed Alomi, Bsc. Pharm, Msc. Clin pharm, BCPS, BCNSP, DiBA, CDE Critical Care Clinical Pharmacists, TPN Clinical Pharmacist, Freelancer Business Planner, Content Editor and Data Analyst, P.O.BOX 100, Riyadh 11392, Riyadh, SAUDI ARABIA.

Phone no: +966 504417712

E-mail:yalomi@gmail.com
Received: 17-12-2020;

Accepted: 05-02-2021;

Copyright: () the author(s), publisher and licensee International Journal of Pharmacology and Clinical Sciences. This is an open-access article distributed under the terms of the Creative Commons

Attribution Non-Commercial License, which permits unrestricted non-commercial use, distribution, and reproduction in any medium, provided the original work is properly cited.

This is an open access article distributed under the terms of the Creative Commons AttributionNonCommercial-ShareAlike 4.0 License

\section{Access this article online}

\begin{tabular}{|c|c|}
\hline & www.ijpcs.net \\
\hline & DOI: \\
\hline &
\end{tabular}

\begin{abstract}
Objectives: To explore the services provided by the forensic pharmacy as the new initiative in the Kingdom of Saudi Arabia. Methods: This is a new project derived from the national and international forensic pharmacy services guidelines. The project has been formulated from the global business model, which is the pharmacy project guidelines of the new project. The initial project is written by the project management professional. It consisted of the initial and planning phase, the execution phase, and the monitoring and controlling phase. Results: The scope of forensic pharmacy services was explored with a defined vision, mission, and goals. The services revealed clinical and economic benefits to the patients. The risk management model assures the continuation of the project. Moreover, the monitoring and controlling of the forensic services as declared. Finally, the project's transition from the operation phase to the closing phase has been explored in this study. Conclusion: The forensic pharmacy practice is a new initiative project, and it was among the pharmacy strategic plan. The forensic pharmacy services were found to meet the requirement of the forensic pharmacy workforces, overcome the requirement of pharmacy services, and improve forensic clinical pharmacy staff. Therefore, it highly suggests implementing forensic pharmacy services in Saudi Arabia.
\end{abstract}

Key words: Forensic pharmacy, Practice, Services, Initiative, Saudi Arabia.

\section{INTRODUCTION}

Pharmacists engage in many activities related to the practice of pharmacy. However, they play a crucial role in maintaining patient safety and health. The last century has witnessed with a remarkable development in various fields related to medicine. However, forensic pharmacy is essential to achieve balance, scientific expansion, and the need for this field with Saudi Vision 2030 programs. Until recently, pharmacists are experts in the science of drugs. Forensic pharmacists apply scientific principles in legal matters and engage in professional work regarding litigation, regulatory procedures, and the criminal justice system. ${ }^{[1]}$ Almost all professions feature a forensic application. ${ }^{[2]}$ The scope of forensic pharmacy is diverse. For instance, there can be a forensic psychiatric pharmacist, a forensic clinical pharmacist, a forensic oncology pharmacist, or a forensic cardiology pharmacist. ${ }^{[3]}$ Moreover, forensic pharmacy plays a significant role in society. It is a specialty that can assist attorneys in case review, the invention process, dispositions, and numerous other kinds of civil and criminal cases. ${ }^{[4]}$ There are no specific training programs or certifications available for forensic pharmacists. However, pharmacists have training in therapeutics, chemistry, pharmacology, pharmacokinetics, and toxicology. All of these areas are often applied to a variety of forensic situations.

The duties of a forensic pharmacist technician include the recommendations and directions of the forensic pharmacist and performs some steps to prepare in advance for the packaging. In addition, they store the chemical reagents and biological materials safely and prepare the necessary radiopharmaceutical materials under the supervision of a forensic pharmacist.

The forensic pharmacist plays a vital role in a wide range of criminal cases, including the analysis, assessment, review, and support of the position of the nuclear physicians. Moreover, the forensic pharmacist disseminates pharmacokinetics, pharmacodynamics, drug interactions, and adverse reactions to detect drug-related topics of radiopharmaceutical products to healthcare professionals. Furthermore, it provides forensic administrative services, including employment testing and doping for athletes. In addition, some distribution services include adulteration or contamination of drugs and the use of counterfeit medicines. Finally, it has an essential role in spreading awareness about evaluation, diagnosis, treatment, and prevention of toxic exposures. ${ }^{[5,6]}$ The forensic clinical pharmacist is responsible for the clinical evaluations of the inappropriate use of chemical agents, adverse drug reactions, date rape drugs, drugged and drunken driving, drug-induced violence, drug-drug interactions, impaired capacity, medication errors, poisoning, or substance abuse.

\section{METHOD OF THE PROJECT}

This study was driven by the international and national forensic pharmacy practice guidelines. ${ }^{[7-13]}$ The task force team of forensic pharmacy practice 
services has been formulated, consisting of the author's expertise in pharmacy administration and clinical pharmacy practitioner. The committee unitized and drove the nuclear pharmacy practice services guidelines from the international literature of forensic pharmacy practice services. It was written by utilizing the global business model, pharmacy project guidelines, and project management institution guidelines of a new project. ${ }^{[14-17]}$ The forensic pharmacy practice services were adjusted based on the types of forensic pharmacy practices, general regulations, and the transformation to the forensic pharmacy practice. The project is done through project management professionals, which consisted of the initial phase, the planning phase, the execution phase, and the monitoring and controlling phase.

\section{Market Analysis}

Forensic medicine services are well established in the Kingdom of Saudi Arabia. These services provide assessment and diagnosis of criminal cases for related reasons. Moreover, toxicological analysis of the material is utilized with forensic medical services. The role of the clinical pharmacist or distributive pharmacist, or pharmacy technician is not clear yet. The pharmacist can provide various services, including but not limited to vigilance drugrelated death, death due to fake medications, medications errors or adverse drug reactions, or serious drug-drug interactions leading to death. Moreover, services, chemical material or cosmetic products, intentional poisoning, drug testing for Olympic new employment services. Pharmacists seldom work in forensic medicine. Unfortunately, there is nil of education and training about forensic applications in pharmacy practice. The opening of new pharmacy services will open new pharmacists jobs and more utilization of pharmacy staff that support new Saudi vision 2030. ${ }^{18}$

\section{Planning phase \\ Scope of the project}

The project covers forensic pharmacy services. It does not include any medications, violent death or death by international poising or suicide death, or any drug-related problem leading to death. However, it has fake or contaminated medicine leading to death, laboratory analysis of any violent medications, analyzing drugrelated proof and criminal behavior, use of drugs or alcohol in automobile accidents or violent actions, legal and illegal pharmaceutical evidence in criminal investigations.

\section{Vision, Missions, and Goals}

The project's vision is to reach the best forensic pharmacy services, whereas the mission is to provide the appropriate forensic pharmacy services for governmental and nongovernmental requirements. The goals of this project were to fix the forensic pharmacy services during transformations to privatization, to establish forensic pharmacy services through involving the pharmacist with Forensic medicine services, and to explore the pharmacist's role in forensic medicine, and educate the population about forensic pharmacy and violent medications with related issues.

\section{Project description}

The following policies and procedures were put in place for every pharmacy staff working at forensic pharmacy services individuals ${ }^{[19]}$

- Forensic pharmacy services should be formulated at all healthcare organizations.

- The forensic pharmacy services committee should consist of the forensic pharmacist, forensic clinical pharmacist, forensic medicine physician, units, pharmacy quality management, medications safety pharmacist, and forensic nurse representative.

- The committee should revise the set standards of the forensic pharmacy services and update them at least annually.

- The committee should conduct education and training sessions about forensic pharmacy services for all pharmacists and healthcare providers.

- The job description The forensic pharmacist and forensic clinical pharmacist should be established.

- Forensic pharmacy services need the policy and procedures distributed to healthcare sectors at the organization by the committee.

- The physician makes the prescription based on the forensic pharmacy services.

- The forensic pharmacist and forensic clinical pharmacist should measure the clinical outcome of the forensic pharmacy services.

- The forensic pharmacist and forensic clinical pharmacist should calculate the economic development of forensic pharmacy services.

- The forensic pharmacist and forensic clinical pharmacist should document any cases of violence to the forensic pharmacy services.

\section{Plan cost management}

For each new project, forensic pharmacy services, the management team must set out the financial budget, including the cost of forensic pharmacy services educational courses, the value of the management team meeting, and the price of updated references of the forensic pharmacy services. The team must monitor and supervise the budget and manage the budget until the project is finished and switch to the operating system.

\section{Executing phase \\ Management team}

Project management professionals of forensic pharmacy services had various steps. One of the primary steps was the execution phase, which had a team-leading the program or the project forensic pharmacy services from the beginning until becoming one of the operating systems at healthcare institutions. The group consisted of forensic pharmacists, forensic clinical pharmacists, forensic medicine physicians, pharmacy quality management, medication safety pharmacists, and forensic nurse representatives. The team needs to be implemented and followed up on the forensic pharmacy services. In addition, the team should educate and train the pharmacy and healthcare staff about the new forensic pharmacy services and measure the clinical and economic outcomes of the project.

\section{Education and training}

Each current new project, forensic pharmacy services, require special education and training. The current project needs education and training for pharmacy staff, including clinical pharmacists, pharmacists, and pharmacy technicians. The healthcare professionals, including physicians and nurses, need another thorough forensic pharmacy services education and training. In addition, the team management needs to be oriented about the project for all healthcare professionals. The orientation emphasizes any new staff healthcare providers joined in the healthcare institutions for forensic pharmacy services.

\section{Project total quality management}

There are various tools for total quantity management, with this project focuses on forensic pharmacy services, emphasizing the implementation phase of the project and project impact. The balance scored cards was among them. ${ }^{[20]}$ The monitoring tools consisted of four parts: the customer, finance, internal process, and education and innovation. The assessment of healthcare services of forensic pharmacy services was an example of an internal-type process. The clinical outcome of forensic pharmacy services might reflect the education and competency of all pharmacy staff education. The financial section is another example of measuring the cost avoidance of forensic pharmacy services. The fourth 
type was the customer-type measuring the patient satisfaction with healthcare providers, including healthcare professionals and pharmacists and pharmacy technicians of forensic pharmacy services satisfaction in Saudi Arabia.

\section{Risk Management}

The project is mainly exposed to risks such as personnel, budget, technical, and quality risks. There are multiple risks: schedule, scope, budget, personal, technological, and quality risks. ${ }^{[21,22]}$ Forensic pharmacy services suffer from personal threats with untrained healthcare professionals or sufficient clinical pharmacists or distributive pharmacists and pharmacy technicians. The budget risk is when the forensic pharmacy services education and training courses are not covered for all pharmacy staff and healthcare professionals. Another technical risk exposed in practice is limited to electronic recourses or unfriendly reporting systems. Finally, forensic pharmacy services might be exposed to quality risks without implementing safety tools or untrained personnel.

\section{Closing of the project}

The forensic pharmacy services across all healthcare institutions of governmental and private sectors are required to declare the role of the forensic pharmacist to prevent drugrelated related issues, morbidity, and mortality. In addition, to avoid an economic burden on the pharmacy and healthcare system, the hospitals and primary healthcare centers should include forensic pharmacy services in Saudi Arabia. The project should continue at the forensic pharmacy services at each healthcare organization. The forensic pharmacy services education and training should be implemented accordingly. Forensic pharmacy services should regularly update and expand the number of pharmacy services recommended in the future. The annual celebration of all forensic pharmacy services staff, including a clinical pharmacist, distributive pharmacist, and pharmacy technician is highly recommended in Saudi Arabia.

\section{ACKNOWLEDGEMENT}

None.

\section{CONFLICT OF INTEREST}

The authors declare that there is no conflict of interest.

\section{Funding}

None

\section{Consent for Publications}

Informed consent was obtained from all the participants

\section{Ethical Approval}

This research was exempted from research and ethical committee or an institutional review board (IRB) approval.

https://www.hhs.gov/ohrp/regulations-andpolicy/decision-charts-2018/index.html

\section{ABBREVIATIONS}

KSA: Kingdom of Saudi Arabia; SWOT: Strengths, Weaknesses, Opportunities and Threats; BSC: Balance Scored Cards.

\section{ORCID ID}

Yousef Ahmed Alomi https://orcid.org/ 0000-0003-1381-628X

\section{REFERENCES}

1. Wick JY. Forensic pharmacy: can you prove it? [internet]. Consult Pharm. 2013;28(7):418-24. doi: 10.4140/TCP.n.2013.418, PMID 23835459.

2. What is forensic science | what is a forensic. Scientist.

3. Kshemada K Kartha CC, Mehta JL. Forensic sciences and growth of cardiology. J Forensic Res. 2013;5(1):115

4. Roles of forensic pharmacy - Docsity [internet] [cited Jun 26 2021]. Available from: https:// www.docsity.com/en/roles-of-forensic-pharmacy/ $5385561 \%$

5. Malve HO. Forensic pharmacology: an important and evolving subspecialty needs recognition in India [internet]. J Pharm Bioallied Sci. 2016;8(2):92-7. doi: 10.4103/0975-7406.171698, PMID 27134459. Available from: /pmc/articles/ PMC4832912.

6. What is a forensic pharmacist? [internet] [cited Jun 26 2021]. Available from: https://work.chron. com/forensic-pharmacist-6623.html.
7. Anderson PD. An overview of forensic pharmacist's practice. J Pharm Pract. 2000;13(3):179-86.

8. Anderson PD. The broad field of forensic pharmacy. J Pharm Pract. 2012;25(1):7-12. doi: 10.1177/0897190011431144, PMID 22251569.

9. Anderson PD, O'Donnell JT. Specific forensic applications for pharmacists. J Pharm Pract. 2000;13(3):187-93. doi: 10.1177/ 089719000001300305

10. Gibler B, Hayes G, Raleigh F, Levenson B, Heber S, Tham A. Forensic psychiatric pharmacy practice at Atascadero State hospital. J Pharm Pract. 1996;9(4):222-8. doi: 10.1177/ 089719009600900403

11. Anderson PD, Naik K, Kinemond C, ImObersteg A Forensic testing for drugs of abuse. J Pharm Pract. 2000;13(3):226-35

12. Yoshizuka KI, Perry PJ. The association between pharmacologic drug intoxication and forensicspecific intent. J Pharm Pract. 2012:25(1):50-60. doi: 10.1177/0897190011431147, PMID 22318915.

13. Anderson PD, Bokor G. Forensic aspects of druginduced violence. J Pharm Pract. 2012;25(1):41-9 doi: 10.1177/0897190011431150, PMID 22215647.

14. McDonough R. Writing a business plan for a new Pharmacy Service. Health. 2010;23, The Dynamics of Pharmaceutical Care: Enriching Patients'.

15. American College of Clinical Pharmacy, Harris IM Baker E, Berry TM, Halloran MA, Lindauer $K$ Ragucci KR, McGivney MS, Taylor AT, Haines ST Developing a business-practice model for pharmacy services in ambulatory settings. Pharmacotherapy. 2008;28(2):285. doi: 10.1592/ phco.28.2.285. PMID 18225974.

16. Sachdev G. Sustainable business models: systematic approach toward successful ambulatory care pharmacy practice. Am J Health Syst Pharm. 2014;71(16):1366-74. doi: 10.2146/ajhp140078, PMID 25074956.

17. $P M B O K$ guide. A guide to the project management body of knowledge. Sixth edit. Project Management Institute, Inc; 2017.

18. Government of Saudi Arabia. Saudi Arabia Vision 2030 [Internet]. 2016. Available from: https://vision2030.gov.sa/sites/default/files/report/Saudi_ Vision2030_EN_2017.pdf

19. Anderson PD. An overview of forensic pharmacists practice. J Pharm Pract. 2000;13(3):179-86.

20. Kaplan RS, Norton DP. The balanced scorecard: Measures That drive performance [Internet]. Vol. 83, Harvard Business Review. 2005 [cited 2020 Mar 15]. Available from: https://hbr.org/1992/01/ the-balanced-scorecard-measures-that-drive-performance-2

21. Ray S. The Risk Management Process in Project Management - ProjectManager.com [Internet]. ProjectManager. 2017 [cited 2020 Mar 15]. Available from: https://www.projectmanager.com/ blog/risk-management-process-steps

22. Kaplan RS, Mikes A. Managing Risks: A New Framework [Internet]. Harvard Business Review. 2012 [cited 2020 Mar 15]. Available from: https:// hbr.org/2012/06/managing-risks-a-new-framework 